\title{
Stimulation Strategies for Tinnitus Suppression in a Neuron Model
}

\author{
Alessandra Paffi $(\mathbb{D}$, Francesca Camera $(\mathbb{D}$, Chiara Carocci, \\ Francesca Apollonio $(\mathbb{D}$, and Micaela Liberti
}

Sapienza University of Rome, Via Eudossiana 18, 00184 Rome, Italy

Correspondence should be addressed to Micaela Liberti; liberti@diet.uniromal.it

Received 29 December 2017; Revised 16 April 2018; Accepted 6 June 2018; Published 30 July 2018

Academic Editor: György Thuróczy

Copyright (C) 2018 Alessandra Paffi et al. This is an open access article distributed under the Creative Commons Attribution License, which permits unrestricted use, distribution, and reproduction in any medium, provided the original work is properly cited.

Tinnitus is a debilitating perception of sound in the absence of external auditory stimuli. It may have either a central or a peripheral origin in the cochlea. Experimental studies evidenced that an electrical stimulation of peripheral auditory fibers may alleviate symptoms but the underlying mechanisms are still unknown. In this work, a stochastic neuron model is used, that mimics an auditory fiber affected by tinnitus, to check the effects, in terms of firing reduction, of different kinds of electric stimulations, i.e., continuous wave signals and white Gaussian noise. Results show that both white Gaussian noise and continuous waves at tens of $\mathrm{kHz}$ induce a neuronal firing reduction; however, for the same amplitude of fluctuations, Gaussian noise is more efficient than continuous waves. When contemporary applied, signal and noise exhibit a cooperative effect in retrieving neuronal firing to physiological values. These results are a proof of concept that a combination of signal and noise could be delivered through cochlear prosthesis for tinnitus suppression.

\section{Introduction}

Tinnitus is a debilitating perception of sound in the absence of external auditory stimuli that affects more than $10 \%$ of the world population [1-3] and tends to increase with the age [2, 3].

The origin of this debilitating disorder may be central or peripheral; i.e., it can originate in the cochlea, in the primary hearing cortex or in any other point of the auditory pathway [4]

Based on frequency and permanence of sound perception, tinnitus is classified in continuous low frequency tinnitus (CLFT) for frequencies below $100 \mathrm{~Hz}$, continuous high frequency tinnitus (CHFT) for frequencies above $3 \mathrm{kHz}$, and transient spontaneous tinnitus (TST) [5]. Several studies $[6,7]$ confirm that the CHFT is the most widespread tinnitus typology, generally associated with a reduction of cochlear functionality at high frequency, due to a damage of the basal section of the cochlea. In the tonotopic organization of sound perception [8], the cochlea basal section encodes for high frequency stimuli, above $3 \mathrm{kHz}$.
This close association between tinnitus and hearing loss suggests that, in many cases, it is due to an impairment of the outer hair cells (OHC) of the cochlear basal section that, in turn, induces a pathologic state of depolarization of the inner hair cells (IHC) [9].

In 1995 Le Page [9] proposed a cochlear model to explain tinnitus origin. The OHCs determine the hair deflection of the IHCs that, in turn, depolarize the acoustic fibers. In physiologic conditions, in the absence of an external stimulus, the OHCs fix the operating point on the IHC transfer function (acoustic neuron depolarization versus IHC hair deflection) to a position that brain recognizes as absence of sound. When the OHCs are damaged, the control input to the IHCs gets lost with a consequent shift of the operating IHC point and a permanent firing rate of the acoustic fiber interpreted by the brain as a real acoustic pattern [9].

This modification of the nerve fiber firing pattern due to OHC impairment was experimentally observed in different animal models [10-13].

Several experimental studies [14-16] revealed that an electric stimulation of the cochlea, delivered through cochlear 
prosthesis or transtympanic electrode, could alleviate tinnitus perception in a significant percentage of treated patients. McKerrow and colleagues [14] used continuous wave (CW) high frequency signals $(2-6 \mathrm{MHz})$ superposed to a Gaussian white noise $(\mathrm{GWN})$, whereas other authors used pulse trains with repetition frequency up to $5 \mathrm{kHz}[15,17]$. Recently, Tyler and colleagues [18] efficiently used pulsed modulated signals delivered to the Vagus nerve on human volunteers.

However, the electric signals delivered in stimulation, in terms of type (CW, pulse train, white noise), frequency content, amplitude, and modulation, were empirically chosen and their mechanisms of action on the auditory fibers were not defined.

Moving from a recent study by the authors [19] showing an inhibitory effect of an electric exogenous stimulation on a hyperexcited neuronal network model, it was hypothesized that an electric stimulation may interfere with the neuron firing pattern of a pathologically polarized acoustic neuron by reducing its firing rate to the physiologic one.

Aim of this work is to verify such a hypothesis and to study the efficacy of a combination of signal and noise in tinnitus inhibition, using a simple model of a hyperexcited auditory fiber.

In a biomedical perspective, the final aim is to deliver this stimulation to the auditory nerve using cochlear prosthesis to suppress tinnitus in patients with acoustic impairment.

\section{Models and Methods}

2.1. Neuron Model. To describe the single Ranvier node of an auditory fiber, a stochastic Hodgkin-Huxley ( $\mathrm{HH}$ ) model was used [20-22]. In this model, the neuronal membrane patch is represented by an electrical equivalent, in which the balance of the currents per unit area is given by

$$
\begin{aligned}
C_{m} \frac{d V}{d t}= & -g_{l}\left(V-E_{l}\right)-g_{K}\left(V-E_{K}\right) \\
& -g_{N a}\left(V-E_{N a}\right)+I_{0}
\end{aligned}
$$

where $C_{m}$ is the unit area capacitance that takes into account the dielectric properties of the membrane phospholipidic bilayer, $V$ is the transmembrane potential, $g_{N a}, g_{K}, g_{l}$ are sodium, potassium and leakage conductances per unit area, respectively, and $E_{N a}, E_{K}, E_{l}$ are the reversal potentials of the corresponding current densities. Finally, $I_{0}$ is the bias current density that controls the transition between the resting state and the firing activity of the neuron [23]. For the deterministic $\mathrm{HH}$ model at $6.3^{\circ} \mathrm{C}$, the threshold value above which the neuron starts its firing activity is equal to $6.3 \mu \mathrm{A} / \mathrm{cm}^{2}[23]$.

Despite the model limitation concerning the operating temperature equal to $6.3^{\circ} \mathrm{C}$, it is simple, very well characterized in terms of neuronal response as a function of model parameters, and the most used in different applications, with more than 10000 citations in the Scopus database [24], so that it can be considered as a golden standard when a new hypothesis has to be tested. Moreover, the possibility of including channel gating stochasticity allowed us to realistically model channel noise which is particularly relevant in the auditory fibers, due to their small size $[25,26]$.

To account for the random gating of sodium and potassium channels, the ionic current densities $I_{N a}=g_{N a}\left(V-E_{N a}\right)$ and $I_{K}=g_{K}\left(V-E_{K}\right)$ were calculated using a channel-statetracking algorithm [27, 28] where Markov chains [27, 29] modeled independent gating particles belonging to each ionic channel.

The magnitude of fluctuations in current densities (channel noise) depends on the number of ionic channels and, thus, for fixed channel densities $\left(\rho_{\mathrm{Na}}=60\right.$ channels $/ \mu \mathrm{m}^{2}$, $\left.\rho_{\mathrm{K}}=18 \mathrm{channels} / \mu \mathrm{m}^{2}\right)$, on the area of the considered membrane patch. Specifically, channel noise is inversely proportional to the square root of the number of ionic channels in the membrane patch [21,30]. Acoustic fibers are characterized by small Ranvier nodes, whose size may vary from 2.2 [25] to $15.7 \mu \mathrm{m}^{2}$ [26] and thus by high levels of intrinsic channel noise. In this work, three patch areas were considered: $2.2,11.0$, and $15.7 \mu \mathrm{m}^{2}$, corresponding to the maximum, the minimum, and an intermediate fiber size.

Besides $\mathrm{Na}, \mathrm{K}$, and leakage current densities, $I_{0}$ represents here the background level of stimulation coming from the OHCs. This current density determines the firing rate of the neuron, i.e., the operating point on the IHC transfer function.

To simulate different states of pathologic neuron depolarization, $I_{0}$ was set to a value close to the threshold: $6 \mu \mathrm{A} / \mathrm{cm}^{2}$ and to suprathreshold values: 7 and $10 \mu \mathrm{A} / \mathrm{cm}^{2}$ [23]. Conversely, physiological spontaneous firing of the auditory fiber was modeled by using a subthreshold bias current density $I_{0}$ equal to $2 \mu \mathrm{A} / \mathrm{cm}^{2}$. With respect to this physiological condition, the other conditions increased the background firing activity from 30 to $80 \%$, as suggested by experimental recordings in animals with induced tinnitus $[12,13]$.

In this paper, for each patch area, four bias currents densities were used: $2,6,7$, and $10 \mu \mathrm{A} / \mathrm{cm}^{2}$. The first value was used to model a healthy acoustic fiber; the other ones modeled paroxysmal excitation underlying tinnitus.

The model was run in the $\mathrm{C}++$ environment using the forward Euler integration method with time step $10 \mu \mathrm{s}$.

In principle, the $\mathrm{HH}$ model extends its validity up to frequencies that short-circuit the membrane capacitance. According to [31], this occurs above the beta relaxation frequency of the cell membrane, at about $100 \mathrm{MHz}$. Moreover, the ionic channel modeling using Markov chains [32] is valid if the sampling time is much longer than the channel protein transition time (order of ps) [33]. The used time step of $10 \mu \mathrm{s}$ imposes a practical limitation of $50 \mathrm{kHz}$ to the maximum frequencies that can be studied with the model. This is well below the theoretical frequency limitations of the model previously discussed.

For each studied condition, 300 independent runs of the model, $1 \mathrm{~s}$ in duration, were considered. The number of runs was approximately the number of afferent fibers contemporary stimulated by a single electrode of the cochlear prosthesis; this number was calculated by considering the size of the electrode $(0.3 \mathrm{~mm})$, the diameter of a IHC $(\approx 10 \mu \mathrm{m})$, and the number of auditory fibers $(\approx 10)$ contacting a single IHC. 
TABLE 1: Mean firing rate (spikes/s) exhibited by the neuron model for different bias current densities $I_{0}$ and patch areas in the absence of external electric stimulation.

\begin{tabular}{lcccc}
\hline & $\begin{array}{c}\text { Sub-threshold } \\
\text { (physiologic) }\end{array}$ & $\begin{array}{c}\text { Close to threshold } \\
\text { (pathologic) }\end{array}$ & $\begin{array}{c}\text { Supra- } \\
\text { threshold } \text { (pathologic) }\end{array}$ \\
\hline Patch area $\left(\mu \mathrm{m}^{2}\right)$ & $I_{0}=2 \mu \mathrm{A} / \mathrm{cm}^{2}$ & $I_{0}=6 \mu \mathrm{A} / \mathrm{cm}^{2}$ & $I_{0}=7 \mu \mathrm{A} / \mathrm{cm}^{2}$ & $I_{0}=10 \mu \mathrm{A} / \mathrm{cm}^{2}$ \\
\hline 2.2 & 53.7 & 64.9 & 66.8 & 72.2 \\
\hline 11.0 & 44.5 & 57.8 & 60.7 & 67.2 \\
\hline 15.7 & 42.1 & 56.5 & 59.3 & 66.2 \\
\hline
\end{tabular}

2.2. Stimulation. The exogenous stimulation was introduced in the model as an additional voltage over the membrane potential [34-36]. In terms of equivalent $\mathrm{HH}$ electric circuit, the electric stimulus was represented as a voltage generator in series with the membrane capacitor and the ionic conductances per unit area [37-40].

The applied electric stimulation was either a $\mathrm{CW}$ or a zero-mean GWN or a combination of both.

It should be noticed that the $\mathrm{CW}$ is a deterministic signal completely characterized by amplitude (A) and frequency (f), whereas the GWN, being a stochastic process, is described by its statistic moments, namely, average value, variance $\left(\sigma_{\mathrm{N}}{ }^{2}\right)$, and autocorrelation function.

The GWN had zero-mean value, flat spectrum, and variance values: ${\sigma_{\mathrm{N}}}^{2}=3,25,100 \mathrm{mV}^{2}$. The variance can be associated with the average power that the process dissipates on a $1 \Omega$ resistance. The $\mathrm{CW}$ signal was chosen to have amplitude values: $\mathrm{A}=1.73,5,10 \mathrm{mV}$, equal to the standard deviations $\left(\sigma_{\mathrm{N}}\right)$ of the considered GWN processes, where $\sigma_{\mathrm{N}}$ was taken as a measure of the amplitude of noise fluctuations. The CW frequencies were chosen to be equal to $25,35,50 \mathrm{kHz}$ because they are above the upper perception threshold of human hearing $(20 \mathrm{kHz})$. Due to the time step of $10 \mu \mathrm{s}$ chosen for the model solution, $50 \mathrm{kHz}$ is the maximum frequency allowed for an input signal. For the same reason, even the GWN spectrum is practically limited to that upper frequency.

After separately studying the two kinds of stimulation, all combinations of the CW signals and the GWN were applied to the model to check possible cooperative effects.

2.3. Quantification of Firing Reduction. As already mentioned in Introduction, a pathologic acoustic fiber exhibits a spontaneous firing rate higher than that of a healthy neuron $[12,13]$. The mean firing rate, i.e., the number of spikes per second, is due to the operating point fixed by the $\mathrm{OHC}$ and to the endogenous noise related to the number of ionic channels. To quantify the level of firing inhibition, and thus of tinnitus suppression, induced by the electric stimulation, it is necessary to introduce a sensitive technique.

In this work, the inactivation function (IA) was defined as follows:

$$
I A=\frac{\# \text { spike }\left(\sigma_{N}=0 ; A=0 ; f=0 ; I_{0}=6,7,10\right)-\# \text { spike }\left(\sigma_{N} \neq 0 ; A \neq 0 ; f \neq 0 ; I_{0}=6,7,10\right)}{\# \text { spike }\left(\sigma_{N}=0 ; A=0 ; f=0 ; I_{0}=6,7,10\right)-\# \text { spike }\left(\sigma_{N}=0 ; A=0 ; f=0 ; I_{0}=2\right)} \times 100
$$

where \#spike $\left(\sigma_{N}=0 ; A=0 ; f=0 ; I_{0}=6,7,10\right)$ is the number of spikes per second of a pathologic neuron $\left(I_{0}=6,7\right.$, $\left.10 \mu \mathrm{A} / \mathrm{cm}^{2}\right)$ in the absence of exogenous electric stimulation $\left(\sigma_{N}=0 \mathrm{mV} ; A=0 \mathrm{mV} ; f=0 \mathrm{~Hz}\right)$; \#spike $\left(\sigma_{N} \neq 0 ; A \neq 0 ; f \neq 0\right.$; $\left.I_{0}=6,7,10\right)$ is the number of spikes per second of a pathologic neuron during the exogenous electric stimulation $\left(\sigma_{N} \neq 0 \mathrm{mV}\right.$; $A \neq 0 \mathrm{mV} ; f \neq 0 \mathrm{~Hz})$; \#spike $\left(\sigma_{N}=0 ; A=0 ; f=0 ; I_{0}=2\right)$ is the number of spikes per second of a healthy neuron $\left(I_{0}=2 \mu \mathrm{A} / \mathrm{cm}^{2}\right)$ in the absence of exogenous electric stimulation $\left(\sigma_{N}=0 \mathrm{mV}\right.$; $A=0 \mathrm{mV} ; f=0 \mathrm{~Hz}$ ).

This quantity furnishes the percentage of firing reduction obtained using the stimulation in the pathologic neuron with respect to the difference, in terms of firing activity, between a pathologic and a physiologic neuron. The inactivation function will be $0 \%$ if the stimulation does not change the number of spikes of pathologic neuron and $100 \%$ if the neuron activity is turned back to the physiologic one. In this latter case, tinnitus is considered completely suppressed. Inactivation could be also higher than $100 \%$ if the firing activity is reduced below the physiologic condition or negative if the effect of electric stimulation is excitatory instead of inhibitory.

\section{Results}

3.1. Spontaneous Firing. The used stochastic neuron model exhibits a firing activity, quantified by the mean firing rate (spikes per second), that increases with the bias current density $I_{0}$ injected in the model, as shown in Table 1 . Even in subthreshold conditions (see second column of Table 1) a not null firing rate is observed, due to the energy injected into the system by channel noise, that increases as the Ranvier node area becomes smaller (Table 1).

The neuron firing rate is due to the contemporary presence of channel noise and bias current density; the first one is determined by the typical sizes of the acoustic Ranvier nodes, the second one accounts for the operating point set by the $\mathrm{OHC}$ on the IHC transfer function, according to [9]. 
As shown in Table 1, for the same patch area, the three bias current densities, used to mimic the neuron with tinnitus (pathologic condition), increase the firing activity with respect to the physiologic condition, here modeled using the subthreshold bias current density $I_{0}=2 \mu \mathrm{A} / \mathrm{cm}^{2}$. These increases range from $21 \%\left(I_{0}=6 \mu \mathrm{A} / \mathrm{cm}^{2}\right)$ to $35 \%$ $\left(I_{0}=10 \mu \mathrm{A} / \mathrm{cm}^{2}\right)$, for the $2.2 \mu \mathrm{m}^{2}$ patch area, from $25 \%$ $\left(I_{0}=6 \mu \mathrm{A} / \mathrm{cm}^{2}\right)$ to $40 \%\left(I_{0}=10 \mu \mathrm{A} / \mathrm{cm}^{2}\right)$, for the $11.0 \mu \mathrm{m}^{2}$ patch area, and from $35 \%\left(I_{0}=6 \mu \mathrm{A} / \mathrm{cm}^{2}\right)$ to $57 \%\left(I_{0}=10 \mu \mathrm{A} / \mathrm{cm}^{2}\right)$, for the $15.7 \mu \mathrm{m}^{2}$ patch area (Table 1). This shows that when channel noise decreases, in correspondence of larger patch areas, bias current densities assume a stronger influence on neuron firing.

The increased firing activity obtained by using the close to threshold and the suprathreshold current densities reported in Table 1 agrees with the experimental recordings on animals with induced tinnitus, reporting an increase from 35 to $83 \%$ $[12,13]$.

In the next sections, it will be examined the efficacy of different exogenous electric stimulations (see Section 2.2) in reducing the firing activity of pathologic neurons down to physiologic conditions.

3.2. Effect of Different Electric Stimulations. The effects of a GWN on the mean firing rate of the neuron model, in each operating condition, have been quantified by the inactivation function IA, defined in Section 2.3, and summarized in Figure 1. For each pathologic condition, Figure 1 shows inactivation versus patch area for three standard deviations $\sigma_{\mathrm{N}}$ of noise fluctuations: $1.73 \mathrm{mV}$ (panel (a)), $5 \mathrm{mV}$ (panel (b)), and $10 \mathrm{mV}$ (panel (c)).

For the lowest $\sigma_{\mathrm{N}}$ (Figure 1(a)), the inactivation does not exceed $2 \%$ and, in some cases, assumes negative values, indicating an increase of the mean firing frequency instead of a reduction. For $\sigma_{\mathrm{N}}$ of $5 \mathrm{mV}$ (Figure 1(b)) it is possible to observe higher inactivation values that increase with the patch area and decrease with the bias current density, reaching a value of about $10 \%$ for patch size $15.7 \mu \mathrm{m}^{2}$ and bias current density $6 \mu \mathrm{A} / \mathrm{cm}^{2}$. However, such values are too low to induce considerable tinnitus alleviation. Further increasing $\sigma_{\mathrm{N}}$ up to $10 \mathrm{mV}$ (Figure $1(\mathrm{c})$ ), the inactivation could become considerable, reaching 53\% for the highest patch area and the smallest bias current density. However, the inactivation is just some percent points for the smallest patch area, where the endogenous channel noise dominates on the exogenous stimulation in determining the neuron firing rate.

Therefore, a standard deviation of $10 \mathrm{mV}$ is necessary for the GWN to induce an inactivation from 26 to $53 \%$ in acoustic fibers whose Ranvier nodes are larger than $11 \mu \mathrm{m}^{2}$.

However, a broadband stimulation with a quite high power, related to the variance of noise fluctuations, may in principle induce unwanted acoustic perceptions coming from neighboring healthy hear cells.

Thus, it is worth evaluating the effect of using a stimulation with comparable amplitude of noise at a single frequency (CW) above $20 \mathrm{kHz}$, the upper perception limit of the human hearing. In fact, this stimulation cannot be directly interpreted as a sound by the human auditory system.
Figure 2(a) shows the inactivation versus the bias current density for the larger patch area (best case) and an applied $\mathrm{CW}$ at $25 \mathrm{kHz}$ and amplitude equal to $1.73,5$, or $10 \mathrm{mV}$. As discussed in Section 2.2, these amplitudes have been chosen to have the same standard deviation of the used GWNs.

Even in this case, the signal with $1.73 \mathrm{mV}$ of amplitude is not efficient in inhibiting firing and that of $5 \mathrm{mV}$ inactivates the neuron up to $10 \%$. The effect becomes considerable for the $10 \mathrm{mV}$ signal, when the inactivation is equal to $18 \%$ for $I_{0}=10 \mu \mathrm{A} / \mathrm{cm}^{2}$ and reaches a maximum of $35 \%$ for $I_{0}=6 \mu \mathrm{A} / \mathrm{cm}^{2}$. As already noticed for the GWN stimulation, the inactivation decreases with the bias current density, i.e., with the background firing activity of the pathologic neuron.

To evaluate the sensitivity to different stimulation frequencies, also 35 and $50 \mathrm{kHz} \mathrm{CW}$ signals have been considered. Figure 2(b) shows the inactivation induced by $25 \mathrm{kHz}$, $35 \mathrm{kHz}$ and $50 \mathrm{kHz} \mathrm{CW}$ signals with the amplitude set to $10 \mathrm{mV}$.

It is worth noticing that the $\mathrm{CW}$ is almost ineffective at $50 \mathrm{kHz}$, being the inactivation always less than $20 \%$, whereas $25 \mathrm{kHz}$ and $35 \mathrm{kHz}$ signals behave in a similar way, with a slightly better performance of the $25 \mathrm{kHz}$ CW. This evidences a frequency sensitivity of the neuron already observed also in a lower frequency range $(50-500 \mathrm{~Hz})[41,42]$.

Results of simulations show that the GWN, having the standard deviation equal to the sinusoidal amplitude, is always more efficient than the $25 \mathrm{kHz} \mathrm{CW}$ in inducing firing reduction. Figure 3 compares the inactivations induced by these two exogenous stimulations in the best case $\left(I_{0}=6 \mu \mathrm{A} / \mathrm{cm}^{2}\right.$; patch area $\left.=15.7 \mu \mathrm{m}^{2}\right)$. Although the inactivation values are very similar when both the noise standard deviation $\left(\sigma_{\mathrm{N}}\right)$ and the signal amplitude $(\mathrm{A})$ are equal to 1.73 and $5 \mathrm{mV}$, for $\sigma_{\mathrm{N}}=10 \mathrm{mV}$ the inactivation induced by GWN is $52 \%$ versus $35 \%$ obtained by using the $25 \mathrm{kHz} \mathrm{CW}$ signal with the same amplitude. In fact, while the $\mathrm{CW}$ inactivation trend versus the amplitude (purple line in Figure 3) is accurately approximated $(\mathrm{R}=0.99976)$ by a quadratic curve with the second-order coefficient equal to 0.35 , in the case of GWN (orange line in Figure 3), the quadratic function which best fits the inactivation trend $(\mathrm{R}=0.99964)$ has a second-order coefficient equal to 0.64 .

To obtain $100 \%$ inactivation, too high amplitude values for the CW signal would be necessary; conversely GWN has the disadvantage of having a spectrum segment in the auditory frequency band.

For these reasons, it would be useful to combine in a suitable way these two kinds of stimulation.

3.3. Effects of Combined Stimulation. The question arises on what happens if monochromatic and white stimulations are combined.

Results of the combined stimulation have been compared to the superposition of the effects induced by the two stimulations applied individually. Figure 4 shows a comparison of inactivation obtained by combining the two kinds of stimulation $\mathrm{IA}(\mathrm{CW}+\mathrm{GWN})$ with the sum of the inactivations obtained by using the two single stimulations 


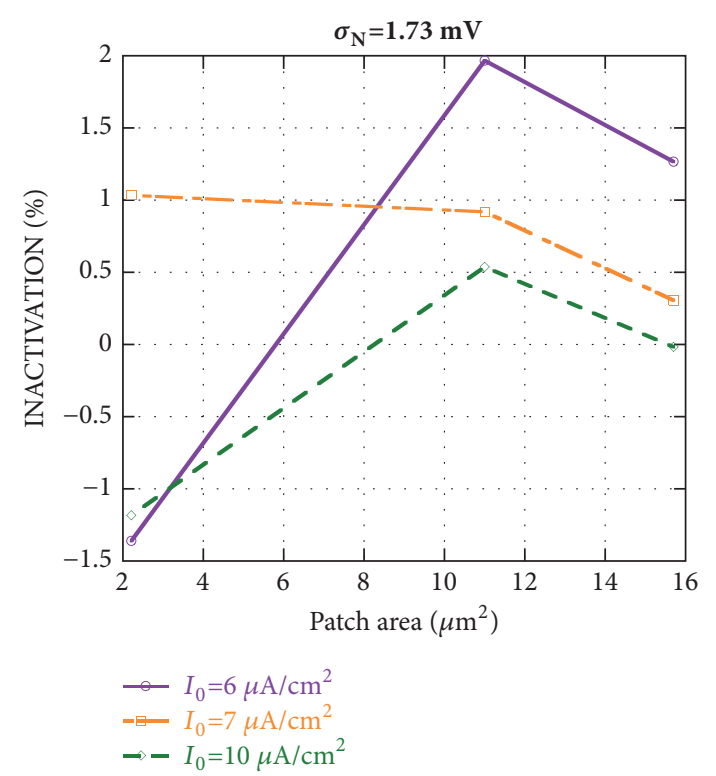

(a)

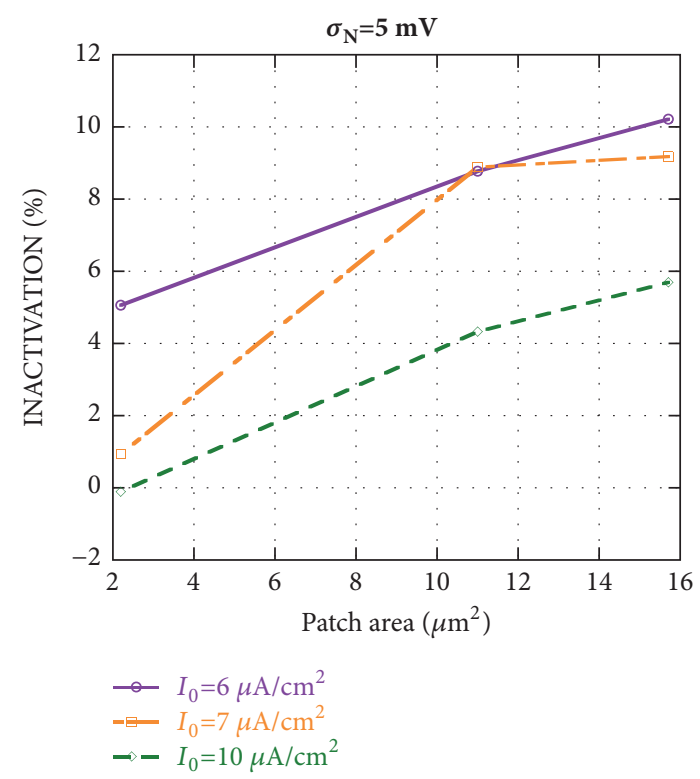

(b)

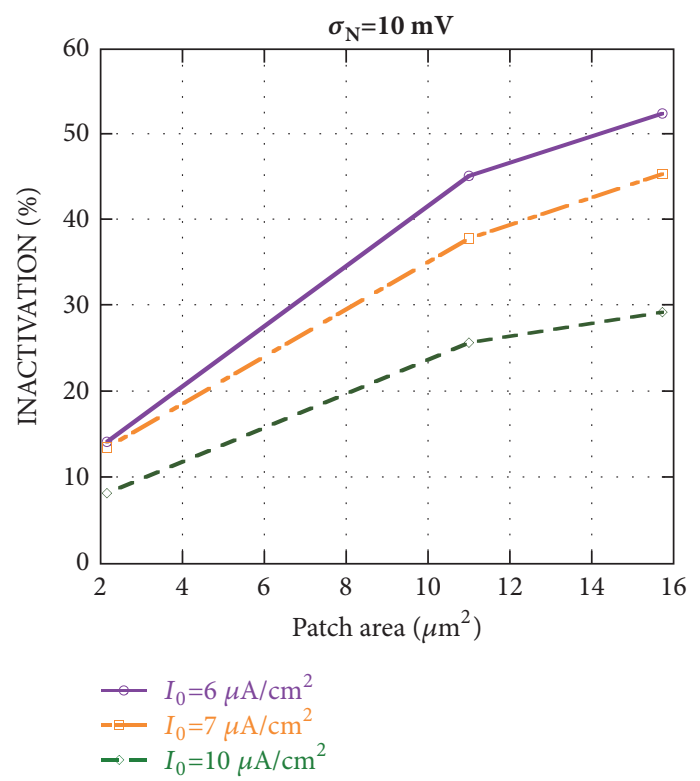

(c)

FIGURE 1: Inactivation versus patch area for different bias current densities $I_{0}$. The exogenous stimulation is given by a Gaussian white noise GWN with different standard deviations: $\sigma_{\mathrm{N}}=1.73 \mathrm{mV}$ (panel (a)), $\sigma_{\mathrm{N}}=5 \mathrm{mV}$ (panel (b)), and $\sigma_{\mathrm{N}}=10 \mathrm{mV}($ panel (c)).

$\mathrm{IA}(\mathrm{CW})+\mathrm{IA}(\mathrm{GWN})$, in the best case: $\mathrm{CW}$ at $25 \mathrm{kHz}$ with amplitude $10 \mathrm{mV}$, and GWN with $\sigma_{\mathrm{N}}=10 \mathrm{mV}$.

As evident from Figure 4, except for the lowest patch area and $I_{0}=6 \mu \mathrm{A} / \mathrm{cm}^{2}, \mathrm{IA}(\mathrm{CW}+\mathrm{GWN})$ is always higher than $\mathrm{IA}(\mathrm{CW})+\mathrm{IA}(\mathrm{GWN})$ and, for $I_{0}=6 \mu \mathrm{A} / \mathrm{cm}^{2}$ and patch area $15.7 \mu \mathrm{m}^{2}$, it reaches $100 \%$. This means that the firing rate of the stimulated neuron is reduced to physiologic conditions.

These results, due to the nonlinear neuronal behavior, show a cooperative effect of the applied signal and noise that can be usefully exploited in applications. So, a good stimulation solution could be a combination of $\mathrm{CW}$ and
GWN to maximize tinnitus suppression while reducing possible side effects.

\section{Discussion}

Results of this work furnish a proof of concept that a suitable exogenous electrical stimulation, consisting of a high frequency $(25-35 \mathrm{kHz}) \mathrm{CW}$ and/or Gaussian noise, can alleviate tinnitus through a mechanism of firing inhibition. This finding is coherent with studies on human volunteers, where the electrical stimulation was delivered to the cochlea [1416], and suggests a possible interaction mechanism based on 


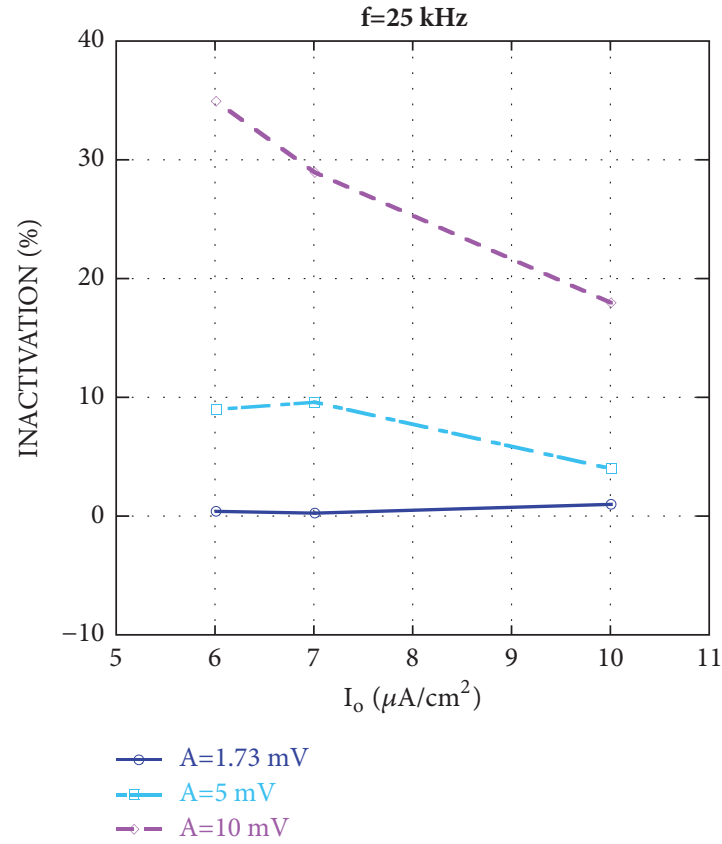

(a)

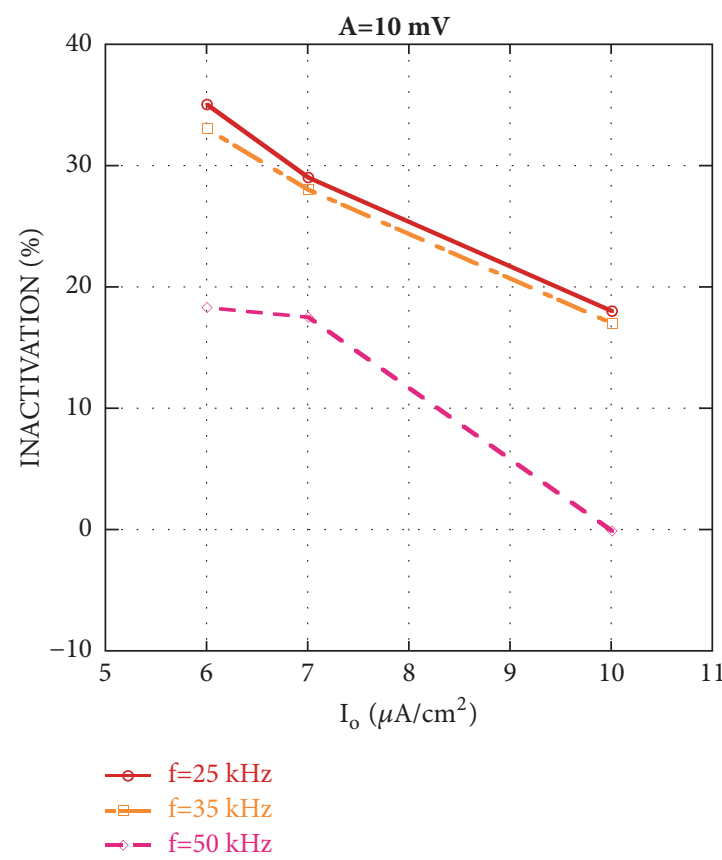

(b)

FIGURE 2: Inactivation versus bias current density $I_{0}$ for a patch area equal to $15.7 \mu \mathrm{m}^{2}$. The exogenous stimulation is given by a CW at $25 \mathrm{kHz}$ and amplitudes $1.73 \mathrm{mV}, 5 \mathrm{mV}$, and $10 \mathrm{mV}$ (panel (a)) or a CW of amplitude $10 \mathrm{mV}$ and frequencies $25 \mathrm{kHz}, 35 \mathrm{kHz}$, and $50 \mathrm{kHz}$ (panel (b)).

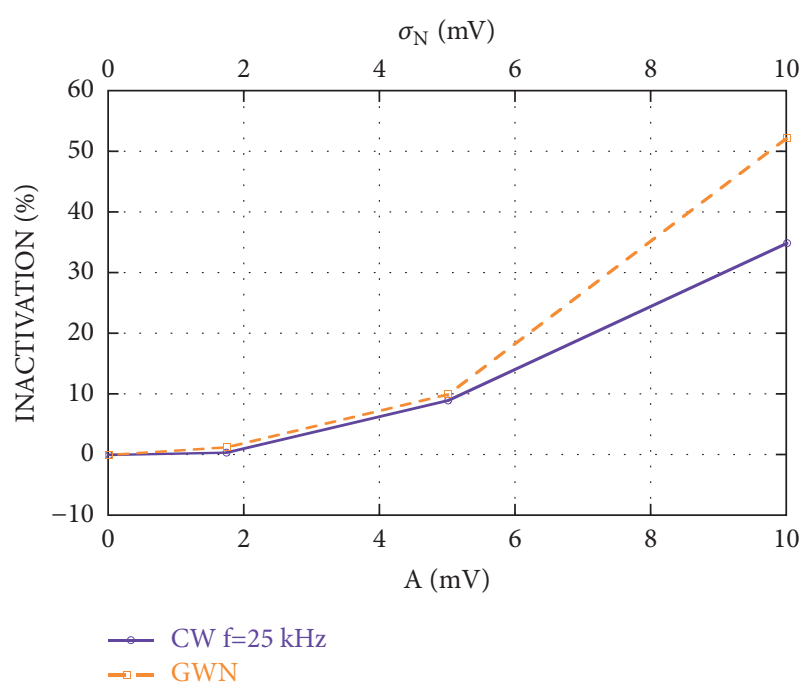

FIgURE 3: Inactivation induced by the $\mathrm{CW}$ stimulation versus the amplitude $\mathrm{A}$ of the $\mathrm{CW}$ at $25 \mathrm{kHz}$ (purple solid line) and inactivation induced by the GWN stimulation versus its standard deviation $\sigma_{\mathrm{N}}$ (orange dashed line); $\mathrm{A}$ and $\sigma_{\mathrm{N}}$ assume the same values; $I_{0}=6 \mu \mathrm{A} / \mathrm{cm}^{2}$; patch area $=15.7 \mu \mathrm{m}^{2}$.

the reduction of the pathologic firing rate to the spontaneous activity of a healthy auditory fiber.

To simulate the single Ranvier node of an auditory fiber, a stochastic $\mathrm{HH}$ neuron model was used, since it is well characterized and considered as a reference model in the literature for a lot of different applications with more than

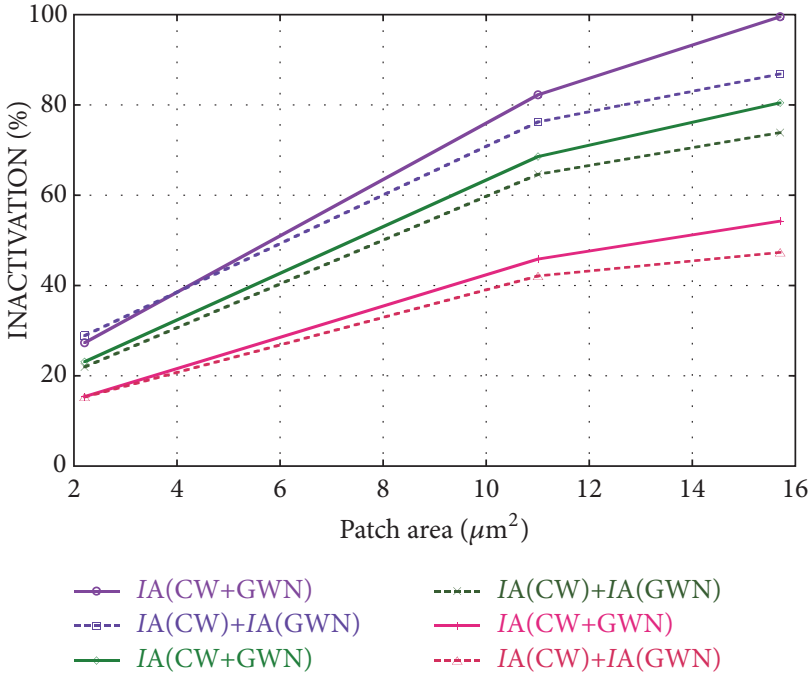

FIGURE 4: Inactivation IA versus patch area for $I_{0}=6 \mu \mathrm{A} / \mathrm{cm}^{2}$ (purple lines), $I_{0}=7 \mu \mathrm{A} / \mathrm{cm}^{2}$ (green lines), and $I_{0}=10 \mu \mathrm{A} / \mathrm{cm}^{2}$ (magenta lines), obtained by combining the $\mathrm{CW}$ at $25 \mathrm{kHz} 10 \mathrm{mV}$ and the $\mathrm{GWN}, \sigma_{\mathrm{N}}=10 \mathrm{mV}$ (solid lines), compared with the superposition of the inactivations induced by the two stimulations applied individually (dashed lines).

10000 citations in the Scopus database [24]. The authors themselves already used it to study neuronal encoding [37, $38,42,43$ ] and to explain the analgesic effect of the Complex Neuroelectromagnetic Pulse [44] by means of a silencing mechanism [19]. 
A limitation of the used model is that, even if a temperature correction factor is used [45], it cannot work at the mammalian temperature of $37^{\circ} \mathrm{C}$. In the $\mathrm{HH}$ model, a temperature increase causes the threshold current density to shift towards higher values, and the firing rate to change depending on the patch size [45]. So, different operating conditions, in terms of bias current densities, would mimic healthy and pathologic neuronal activities. Similar mechanisms of relative firing reduction are expected to occur for a suitable combination of signal and noise since the model anyway presents two attraction basins for firing ad resting states and the exogenous stimulation can push the system from one state to the other. However, since the temperature adjustment in neuronal models is still an open question, here it was preferred to use the well-assessed reference temperature for the $\mathrm{HH}$ model.

Due to the generality of the used model and the high number of degrees of freedom, a complete evaluation of the uncertainty budget is not practicable but, besides the temperature, the other main variables that may influence results are examined in the following.

An aspect that could contribute to the uncertainty of results is that, for frequencies above $10 \mathrm{kHz}$, the membrane capacitance per unit area $\left(\mathrm{C}_{\mathrm{m}}\right)$ is not constant, differently from what was assumed in our model. In fact, the permittivity of the cell membrane decreases with frequency due to the relaxation of the alpha polarization phenomenon [46]. Nevertheless, our simplification is largely acceptable since the frequency dependence of $\mathrm{C}_{\mathrm{m}}$ was shown to have a negligible effect on the stimulation threshold of a $\mathrm{HH}$ model (median = 1.4\%) [47].

Other model parameters that induce a great variability of results are the bias current density $I_{0}$ and the patch area. When applying a combination of the $\mathrm{CW}(\mathrm{f}=25 \mathrm{kHz}$, $\mathrm{A}=10 \mathrm{mV})$ and the GWN $\left(\sigma_{\mathrm{N}}=10 \mathrm{mV}\right)$ to the neuronal patch of $15.7 \mu \mathrm{m}^{2}$, the inactivation ranges from $54 \%\left(I_{0}=10 \mu \mathrm{A} / \mathrm{cm}^{2}\right)$ to $100 \%\left(I_{0}=6 \mu \mathrm{A} / \mathrm{cm}^{2}\right)$. Conversely, for $I_{0}=6 \mu \mathrm{A} / \mathrm{cm}^{2}$, the inactivation passes from $28 \%$ to $100 \%$ if the patch size increases from 2.2 to $15.7 \mu \mathrm{m}^{2}$. Such variations could explain the great variability of results on human volunteers [16] that could be attributed to the individual variability of auditory fiber size (patch area in the model) and tinnitus severity (bias current density in the model).

This study suggests a plausible mechanism of tinnitus suppression using exogenous electrical excitation and is a first step towards the characterization of kind and parameters of stimulation that maximize the efficacy while reducing possible short-term or long-term side effects, such as unwanted sound perception or adaptation.

To control side effects, charge-balanced signals should be used and the induced currents should not exceed typical currents used in cochlear prostheses. A recent dosimetric study [48] revealed that a typical cochlear implant delivered, at the location of the afferent fibers of the auditory nerve, a peak voltage of several tens of $\mathrm{mVs}$, higher than the signal amplitudes used in this work $(\leq 10 \mathrm{mV})$. This suggests that the stimulation signals used in this work are plausible to be released from cochlear implants without severe side effects, even though it will be necessary to conduct a careful risk analysis to assess the safety of the proposed technique.

\section{Conclusions}

A stochastic $\mathrm{HH}$ neuron model was used to evaluate the efficacy of different electric stimulation strategies in tinnitus suppression. The used stimulations were $\mathrm{CW}$ signals at different frequencies in the range of tens of $\mathrm{kHz}$ and GWN.

Results of simulations show that both a CW and a white noise, applied individually to the neuron model, may induce a firing inhibition. The inactivation level is shown to depend on many parameters, such as patch area, bias current density, CW frequency and amplitude, and noise standard deviation. The more the background activity is low (larger patch size and lower bias currents), the more the inactivation is high. Considerable inactivation values are obtained by using either $\mathrm{CW}$ at 25 or $35 \mathrm{kHz}$ or GWN with $10 \mathrm{mV}$ of standard deviation, but GWN is shown to be more efficient than $\mathrm{CW}$ ( $\mathrm{IA}=53 \%$ versus $\mathrm{IA}=35 \%$ in the best condition) for a comparable amplitude of fluctuations.

Moreover, the inactivation induced by a combination of signal and noise is almost always higher than the sum of the inactivations induced by the two stimulations applied individually and it reaches $100 \%$ for the lowest $I_{0}$ and the highest patch area.

These results are a proof of concept that signal and noise act on the neuron in a cooperative way and could be suitably delivered in combination through cochlear prosthesis to alleviate tinnitus while reducing possible side effects due to a broadband stimulation.

Future works will concern the validation of the presented results on a mammalian neuronal model at $37^{\circ} \mathrm{C}$, such as the Spatially Extended Nonlinear Node (SENN) [49] and the McIntyre-Richardson-Grill (MRG) [50] models and the identification of a colored stimulating noise suitably filtered considering the typical frequency selectivity of the used model.

\section{Disclosure}

This work was partially performed within the context of the European COST EMF-MED Action BM1309. Preliminary results were presented at the Joint Annual Meeting of the Bioelectromagnetics Society and the European BioElectromagnetics Association, Ghent, Belgium, 2016.

\section{Conflicts of Interest}

The authors report no conflicts of interest. The authors alone are responsible for the content and writing of the paper.

\section{References}

[1] K. Brunger, "Managing tinnitus.," The Journal of Family Health Care, vol. 18, no. 2, pp. 47-48, 2008.

[2] J. Shargorodsky, G. C. Curhan, and W. R. Farwell, "Prevalence and characteristics of tinnitus among US adults," American Journal of Medicine, vol. 123, no. 8, pp. 711-718, 2010. 
[3] B. Scott and P. Lindberg, "Psychological profile and somatic complaints between help-seeking and non-help-seeking tinnitus subjects," Psychosomatics, vol. 41, no. 4, pp. 347-352, 2000.

[4] H. P. Zenner, M. Pfister, and N. Birbaumer, “Tinnitus sensitization: Sensory and psychophysiological aspects of a new pathway of acquired centralization of chronic tinnitus," Otology \& Neurotology, vol. 27, no. 8, pp. 1054-1063, 2006.

[5] G. Baracca, L. Del Bo, and U. Ambrosetti, “Tinnitus and hearing loss," in Textbook of Tinnitus, A. R. Møller, B. Langguth, D. De Ridder, and T. Kleinjung, Eds., pp. 285-291, Springer, New York, NY, USA, 2011.

[6] C. Nicolas-Puel, T. Akbaraly, R. Lloyd et al., "Characteristics of tinnitus in a population of 555 patients: Specificities of tinnitus induced by noise trauma," International Tinnitus Journal, vol. 12, no. 1, pp. 64-70, 2006.

[7] F. Martines, D. Bentivegna, E. Martines, V. Sciacca, and G. Martinciglio, "Characteristics of tinnitus with or without hearing loss: Clinical observations in Sicilian tinnitus patients," Auris Nasus Larynx, vol. 37, no. 6, pp. 685-693, 2010.

[8] G. Ehret, “Tonotopic organization (maps)," in Encyclopedia of Neuroscience, pp. 4083-4088, 2009.

[9] E. L. Le Page, "A model for cochlear origin of subjective tinnitus: excitatory drift in the operating point of inner hair cells," in Mechanisms of Tinnitus, J. A. Vernon and A. R. Moller, Eds., pp. 115-148, Allyn and Bacon, London, 1995.

[10] A. J. Noreña, M. Tomita, and J. J. Eggermont, "Neural changes in cat auditory cortex after a transient pure-tone trauma," Journal of Neurophysiology, vol. 90, no. 4, pp. 2387-2401, 2003.

[11] A. J. Noreña and J. J. Eggermont, "Changes in spontaneous neural activity immediately after an acoustic trauma: implications for neural correlates of tinnitus," Hearing Research, vol. 183, no. 1-2, pp. 137-153, 2003.

[12] J. A. Kaltenbach, "Tinnitus: models and mechanisms," Hearing Research, vol. 276, no. 1-2, pp. 52-60, 2011.

[13] P. G. Finlayson and J. A. Kaltenbach, "Alterations in the spontaneous discharge patterns of single units in the dorsal cochlear nucleus following intense sound exposure," Hearing Research, vol. 256, no. 1-2, pp. 104-117, 2009.

[14] W. S. Mckerrow, C. E. Schreiner, M. M. Merzenich, R. L. Snyder, and J. G. Toner, "Tinnitus suppression by cochlear implants," Annals of Otology, Rhinology \& Laryngology, vol. 100, no. 7, pp. 552-558, 1991.

[15] J. T. Rubinstein, R. S. Tyler, A. Johnson, and C. J. Brown, "Electrical suppression of tinnitus with high-rate pulse trains," Otology \& Neurotology, vol. 24, no. 3, pp. 478-485, 2003.

[16] J. E. Chang and F. Zeng, "Tinnitus suppression by electric stimulation of the auditory nerve," Frontiers in Systems Neuroscience, vol. 6, article 19, 2012.

[17] F.-G. Zeng, Q. Tang, A. Dimitrijevic, A. Starr, J. Larky, and N. H. Blevins, "Tinnitus suppression by low-rate electric stimulation and its electrophysiological mechanisms," Hearing Research, vol. 277, no. 1-2, pp. 61-66, 2011.

[18] R. Tyler, A. Cacace, C. Stocking et al., "Vagus Nerve Stimulation Paired with Tones for the Treatment of Tinnitus: A Prospective Randomized Double-blind Controlled Pilot Study in Humans," Scientific Reports, vol. 7, no. 1, 2017.

[19] F. Camera, A. Paffi, A. W. Thomas et al., "The CNP signal is able to silence a supra threshold neuronal model," Frontiers in Computational Neuroscience, vol. 9, Article 44, pp. 1-11, 2015.

[20] A. L. Hodgkin and A. F. Huxley, "A quantitative description of membrane current and its application to conduction and excitation in nerve.," The Journal of Physiology, vol. 117, no. 4, pp. 500-544, 1952.

[21] E. Schneidman, B. Freedman, and I. Segev, "Ion Channel Stochasticity May Be Critical in Determining the Reliability and Precision of Spike Timing," Neural Computation, vol. 10, no. 7, pp. 1679-1703, 1998.

[22] J. R. Clay and L. J. DeFelice, "Relationship between membrane excitability and single channel open-close kinetics," Biophysical Journal, vol. 42, no. 2, pp. 151-157, 1983.

[23] E. M. Izhikevich, Dynamical Systems in Neuroscience: The Geometry of Excit- ability and Bursting, MIT Press, Cambridge, UK, 2006.

[24] Scopus database, (https://www.scopus.com).

[25] F. Rattay, "Basics of hearing theory and noise in cochlear implants," Chaos, Solitons \& Fractals, vol. 11, no. 12, pp. 1875$1884,2000$.

[26] H. Mino and W. M. Grill Jr., "Effects of stochastic sodium channels on extracellular excitation of myelinated nerve fibers," IEEE Transactions on Biomedical Engineering, vol. 49, no. 6, pp. 527-532, 2002.

[27] H. Mino, J. T. Rubinstein, and J. A. White, "Comparison of algorithms for the simulation of action potentials with stochastic sodium channels," Annals of Biomedical Engineering, vol. 30, no. 4, pp. 578-587, 2002.

[28] J. H. Goldwyn, N. S. Imennov, M. Famulare, and E. Shea-Brown, "Stochastic differential equation models for ion channel noise in Hodgkin-Huxley neurons," Physical Review E: Statistical, Nonlinear, and Soft Matter Physics, vol. 83, no. 4, 2011.

[29] J. T. Rubinstein, "Threshold fluctuations in an $\mathrm{N}$ sodium channel model of the node of Ranvier," Biophysical Journal, vol. 68, no. 3, pp. 779-785, 1995.

[30] J. A. White, J. T. Rubinstein, and A. R. Kay, "Channel noise in neurons," Trends in Neurosciences, vol. 23, no. 3, pp. 131-137, 2000.

[31] C. Merla, M. Liberti, F. Apollonio, and G. D’inzeo, “Quantitative assessment of dielectric parameters for membrane lipid bilayers from rf permittivity measurements," Bioelectromagnetics, vol. 30, no. 4, pp. 286-298, 2009.

[32] E. Neher and B. Sakmann, "Single-channel currents recorded from membrane of denervated frog muscle fibers," in A Century of Nature: Twenty-One Discoveries that Changed Science and the World, L. Garwin and T. Lincoln, Eds., 2003.

[33] P. Marracino, M. Liberti, E. Trapani et al., "Human aquaporin 4 gating dynamics under perpendicularly-oriented electric-field impulses: A molecular dynamics study," International Journal of Molecular Sciences, vol. 17, no. 7, article no. 1133, 2016.

[34] C. Merla, A. Denzi, A. Paffi et al., "Novel passive element circuits for microdosimetry of nanosecond pulsed electric fields," IEEE Transactions on Biomedical Engineering, vol. 59, no. 8, pp. 23022311, 2012.

[35] F. Apollonio, M. Liberti, A. Paffi et al., "Feasibility for microwaves energy to affect biological systems via nonthermal mechanisms: a systematic approach," IEEE Transactions on Microwave Theory and Techniques, vol. 61, no. 5, pp. 2031-2045, 2013.

[36] A. Denzi, C. Merla, P. Camilleri et al., "Microdosimetric study for nanosecond pulsed electric fields on a cell circuit model with nucleus," Journal of Membrane Biology, vol. 246, no. 10, pp. 761767, 2013.

[37] A. Paffi, F. Apollonio, G. d'Inzeo, and M. Liberti, "Stochastic resonance induced by exogenous noise in a model of a neuronal 
network," Network: Computation in Neural Systems, vol. 24, no. 3, pp. 99-113, 2013.

[38] A. Paffi, F. Camera, F. Apollonio, G. D'Inzeo, and M. Liberti, "Restoring the encoding properties of a stochastic neuron model by an exogenous noise," Frontiers in Computational Neuroscience, vol. 9, Article 42, pp. 1-11, 2015.

[39] T. Y. Tsong and R. D. Astumian, "Electroconformational coupling and membrane protein function," Progress in Biophysics and Molecular Biology, vol. 50, no. 1, pp. 1-45, 1987.

[40] H. Mino, J. T. Rubinstein, C. A. Miller, and P. J. Abbas, "Effects of electrode-to-fiber distance on temporal neural response with electrical stimulation," IEEE Transactions on Biomedical Engineering, vol. 51, no. 1, pp. 13-20, 2004.

[41] F. Liu, J. Wang, and W. Wang, "Frequency sensitivity in weak signal detection," Physical Review E: Statistical, Nonlinear, and Soft Matter Physics, vol. 59, no. 3, pp. 3453-3460, 1999.

[42] S. Orcioni, A. Paffi, F. Camera, F. Apollonio, and M. Liberti, "Automatic decoding of input sinusoidal signal in a neuron model: Improved SNR spectrum by low-pass homomorphic filtering," Neurocomputing, vol. 267, pp. 605-614, 2017.

[43] S. Orcioni, A. Paffi, F. Camera, F. Apollonio, and M. Liberti, "Automatic decoding of input sinusoidal signal in a neuron model: high pass homomorphic filtering," Neurocomputing, vol. 292, pp. 165-173, 2018.

[44] A. W. Thomas, M. Kavaliers, F. S. Prato, and K.-P. Ossenkopp, "Antinociceptive effects of a pulsed magnetic field in the land snail, Cepaea nemoralis," Neuroscience Letters, vol. 222, no. 2, pp. 107-110, 1997.

[45] L. Yang and Y. Jia, "Effects of patch temperature on spontaneous action potential train due to channel fluctuations: Coherence resonance," BioSystems, vol. 81, no. 3, pp. 267-280, 2005.

[46] K. R. Foster and H. P. Schwan, "Dielectric properties of tissues and biological materials: a critical review," Critical Reviews in Biomedical Engineering, vol. 17, pp. 25-104, 1989.

[47] B. Howell, L. E. Medina, and W. M. Grill, "Effects of frequencydependent membrane capacitance on neural excitability," Journal of Neural Engineering, vol. 12, no. 5, Article ID 056015, 2015.

[48] G. Tognola, A. Pesatori, M. Norgia et al., "Numerical modeling and experimental measurements of the electric potential generated by cochlear implants in physiological tissues," IEEE Transactions on Instrumentation and Measurement, vol. 56, no. 1, pp. 187-193, 2007.

[49] J. P. Reilly and A. M. Diamant, Electrostimulation Theory, Applications, and Computational Models, 2011, http://www .artechhouse.com.

[50] C. C. McIntyre, A. G. Richardson, and W. M. Grill, "Modeling the excitability of mammalian nerve fibers: Influence of afterpotentials on the recovery cycle," Journal of Neurophysiology, vol. 87, no. 2, pp. 995-1006, 2002. 


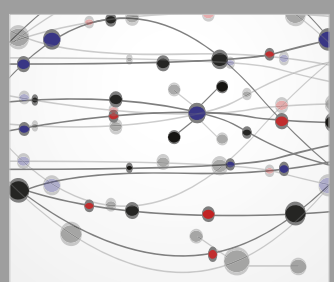

The Scientific World Journal
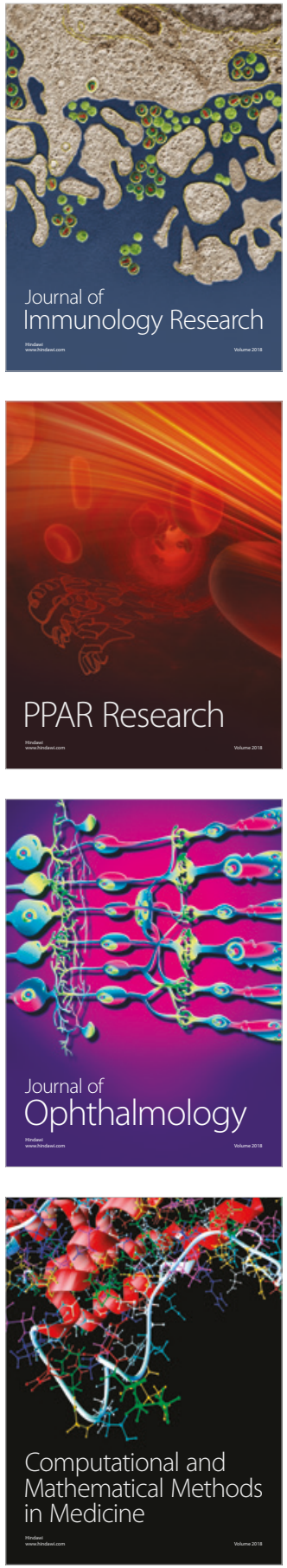

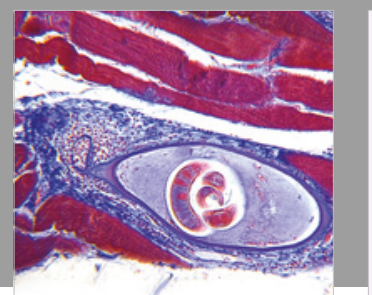

Gastroenterology Research and Practice

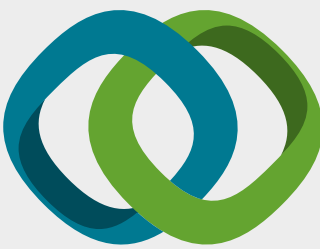

\section{Hindawi}

Submit your manuscripts at

www.hindawi.com
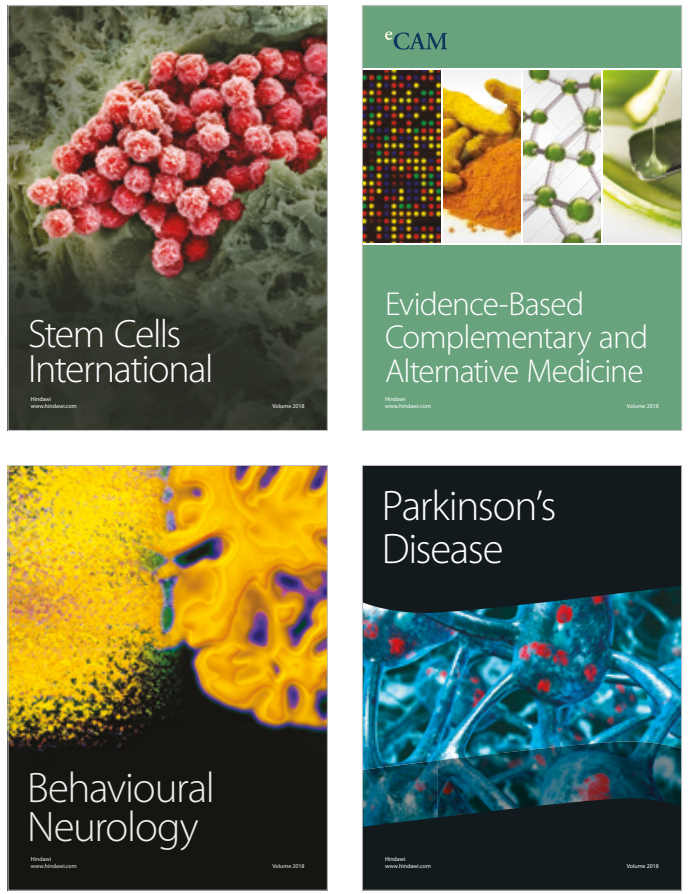

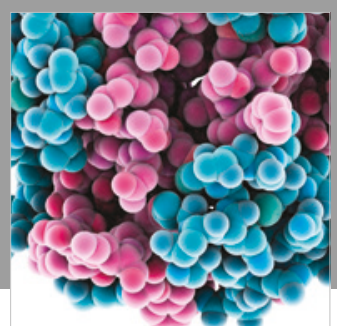

ournal of

Diabetes Research

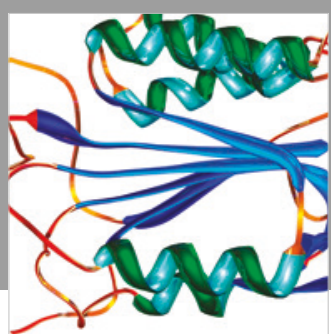

Disease Markers
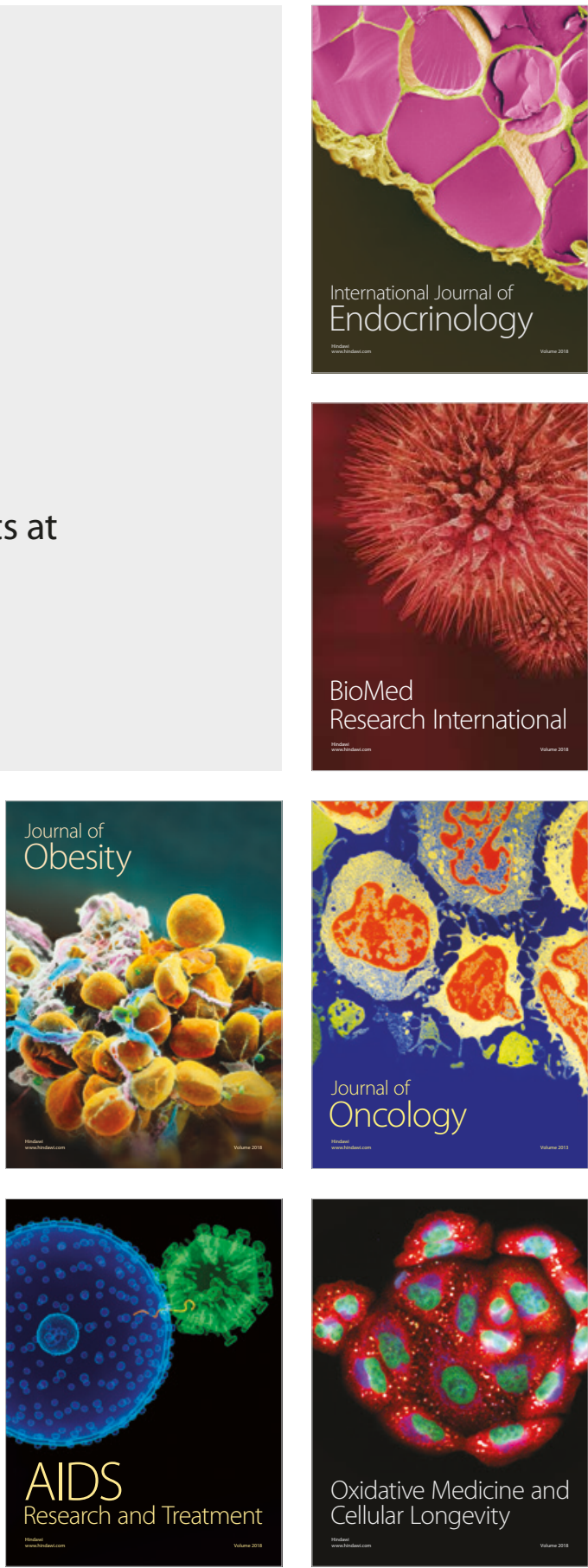